\title{
Scaling Properties and Wave Interactions in Confined Supersonic Turbulent Bluff-Body Wakes
}

\author{
Masaki Nakagawa* and Werner J. A. Dahm ${ }^{\dagger}$ \\ University of Michigan, Ann Arbor, Michigan 48109-2140
}

\begin{abstract}
Results are presented from an experimental study that compares the large-scale structure, mean velocities, and scaling properties of confined, supersonic, planar, turbulent, bluff-body wakes at $M_{\infty} \approx 2$ and 3 with corresponding results from incompressible wakes. The large-scale structure of incompressible planar turbulent wakes is recovered at downstream locations $x$ where the local relative Mach number $M_{r}(x)$ has decreased to about 0.4 . Interactions between these large-scale structures and reflected expansion waves from the near-field recompression region lead to substantial changes in the scaling properties of the flow. Wave interactions with the flow create local self-induced forcing as the large-scale structures pass through the reflected expansion waves. In the $M_{\infty} \approx 2$ wake, a subsonic upstream path exists from the first wave interaction point that allows upstream propagation of the wave-induced forcing, and consequently the measured wake scaling constants match values reported from forced incompressible wakes. In the $M_{\infty} \approx 3$ wake, no subsonic upstream path exists from the first interaction point, and as a consequence the measured scaling constants match values reported from unforced incompressible wakes. Downstream of each wave interaction point, the self-induced forcing leads to an increase in the wake growth rate and in the entrainment of free-stream fluid. The wake subsequently detrains this fluid and returns to its original growth rate. This local forcing mechanism and its effects on the wake scaling properties repeat at each downstream location where the reflected expansion waves intersect the wake.
\end{abstract}

\section{Introduction}

$\mathbf{I}$ $\mathrm{N}$ most supersonic combustors, a subsonic fuel stream must be mixed and burned with a supersonic air stream under conditions that typically correspond to mixing-limited reaction and entrainment-limited mixing. The overall combustion process thus depends on the ability to achieve sufficiently large rates of entrainment of oxidizer into the injected fuel stream. However, compressibility effects in turbulent shear flows are known to reduce the entrainment and mixing rates significantly from those occurring in a corresponding subsonic flow. For this reason, numerous fundamental studies have sought to develop an understanding of the effects of compressibility on entrainment and mixing processes in supersonic turbulent shear flows by studying elementary canonical flows under carefully controlled conditions.

The majority of such studies have addressed entrainment and mixing in supersonic mixing layers, ${ }^{1-20}$ from which several specific effects of compressibility have been observed. These include changes in the scaling properties of the flow, principally a reduction in the growth rate with increasing convective Mach number $M_{c}$ and a corresponding reduction in the entrainment and mixing rates achieved by the flow, as well as changes in the structure and dynamics of the large scales, including a loss of two-dimensional spanwise coherence of large-scale vortical structures. Incompressible planar turbulent wakes also have a strongly pronounced and comparatively well-organized large-scale vortical structure and, like the mixing layer, they follow relatively simple power-law scalings in the entrainment and mixing properties of the flow. Corresponding supersonic planar turbulent wakes are closely related to many

Received 30 August 2005; accepted for publication 21 November 2005. Copyright (C) 2006 by Werner J. A. Dahm. Published by the American Institute of Aeronautics and Astronautics, Inc., with permission. Copies of this paper may be made for personal or internal use, on condition that the copier pay the $\$ 10.00$ per-copy fee to the Copyright Clearance Center, Inc., 222 Rosewood Drive, Danvers, MA 01923; include the code 0001-1452/06 $\$ 10.00$ in correspondence with the CCC.

${ }^{*}$ Graduate Student Research Assistant, Laboratory for Turbulence and Combustion, Department of Aerospace Engineering; currently Research Engineer, Aerodynamics Department, Toyota Motorsport GmBH, Toyota Allee 7, D-50858, Cologne, Germany.

${ }^{\dagger}$ Head, Laboratory for Turbulence and Combustion, Department of Aerospace Engineering. of the flows encountered in practical supersonic combustors. These include supersonic bluff-body wakes, in which the body thickness is much greater than the boundary layer thickness and the flow thus separates from the wake generator. Such supersonic bluff-body wakes create strong expansion and recompression waves in a relatively complicated base flow region, as well as a supersonic wake flow farther downstream in which compressibility effects can play a large role in altering the entrainment and mixing properties relative to the corresponding incompressible wakes.

Most studies of supersonic planar turbulent bluff-body wakes have focused on flow properties in the base flow region. ${ }^{21-25}$ There have been few detailed studies that span from the near-field base flow region well into the self-similar far-field wake. Amatucci et al. ${ }^{26}$ have used two-component laser Doppler velocimetry (LDV) measurements to document profiles of mean velocity and turbulence statistics in the near and far fields of a supersonic bluff-body wake. However, to date no studies have investigated the scaling properties of supersonic turbulent bluff-body wakes, and no detailed comparisons have been made with corresponding incompressible turbulent wakes to determine the effects of compressibility on the entrainment and mixing processes in a manner analogous to that done in supersonic turbulent mixing layers. Moreover, in practical supersonic combustors the presence of sidewalls will lead to reflections of strong shock and expansion waves from the near-field recompression region, which will subsequently intersect the wake. Despite this, no studies have yet examined such effects of confinement on the far-field of supersonic turbulent bluff-body wakes, though resulting wave interactions with the flow may be expected to produce significant changes in the entrainment and mixing properties of confined supersonic bluff-body wakes.

Here we report results from an experimental investigation of confined supersonic, planar, turbulent, bluff-body wakes to help clarify the effects of compressibility on entrainment and mixing in practical supersonic turbulent shear flows. The experiments are based on planar laser Mie scattering (PLMS) and schlieren visualizations of the vortical structure and the growth rate of the flow, together with pressure measurements to determine mean velocity fields and scaling properties of these wakes. The main focus is on identifying scaling properties and scaling constants in the wake far field to discern the effects of compressibility, and on the effects of confinement on these scaling properties. Comparisons are thus presented of scaling properties in confined supersonic turbulent bluff-body wakes 
at freestream Mach numbers $M_{\infty} \approx 2$ and 3 , and these results are thus compared with corresponding results from forced and unforced incompressible turbulent wakes.

\section{Experimental Facility and Diagnostics}

The experiments were conducted in a facility specifically designed for studies of supersonic coflowing turbulent jets and wakes. Figure 1 shows the test section, which has interior cross-sectional dimensions of $34.6 \times 38.4 \mathrm{~mm}$ and is $553 \mathrm{~mm}$ in length. There are eight probe ports on each of the test section sidewalls to allow pressure probe measurements along the transverse direction at several streamwise locations. The test section sidewalls were kept parallel for all results presented here; variations in wall static pressure due to the displacement effect of the wake were negligible in comparison with variations induced by the repeated reflections of the wake expansion and recompression waves from the tunnel sidewalls. The test section has $38.5 \times 229 \mathrm{~mm}$ Vycor windows on each of the front and back walls. Two side windows each have a $7.5 \times 229 \mathrm{~mm}$ viewing area and replace the pressure-port sidewalls to allow access for optical diagnostics.

A subsonic slot nozzle also shown in Fig. 1 was centered in the test section entrance plane, with exterior dimensions of $4.2 \times 38.4 \mathrm{~mm}$ and interior dimensions of $2.0 \times 36.2 \mathrm{~mm}$. This entered the tunnel through a 1/4-in. i.d. round tube and then flattened in the spanwise direction as it extended into the test section $2 \mathrm{~mm}$ past the end of the supersonic nozzle. The slot nozzle was packed with glass beads and screens to produce a uniform low-speed seed flow across the exit plane of the slot, at volume flow rates typically below $1.0 \times 10^{-3} \mathrm{~m}^{3} / \mathrm{s}$. Reynolds numbers $R e_{L}$ based on the length $L$ from the throat of the supersonic nozzle to the end of the slot nozzle were in the range of $3 \times 10^{6}$ to $5 \times 10^{6}$, and thus the boundary layers on the outside of the slot nozzle wall were turbulent. These produced a drag per unit span $D=\rho_{\infty} U_{\infty}^{2} \vartheta_{\mathrm{BL}}$ that greatly exceeded the momentum flux per unit span $J_{0}=\rho_{0} U_{0}^{2} b_{0}$ created by the seed flow through the slot nozzle; here $\vartheta_{\mathrm{BL}}$ is the boundary layer momentum thickness and $b_{0}$ is the width of the slot nozzle. The nozzle thus acted as a bluff-body wake generator, with the seed flow through it allowing visualization of the resulting turbulent wake. The resulting net drag $\left(D-J_{0}\right)=\rho_{\infty} U_{\infty}^{2} \vartheta$ produced an initial wake momentum thickness $\vartheta$. In incompressible wakes without any streamwise any pressure gradient, this initial $\vartheta$ remains constant at all downstream locations. In a confined supersonic wake, the changes in density and velocity produced by wave reflections create changes in the local momentum thickness once the reflected waves from the near-field recompression region intersect the wake. However, upstream of the first wave intersection point, the momentum thickness remains constant at the inital value, here denoted by $\vartheta$. For the Mach 2 and Mach 3 wakes, free stream velocities were $U_{\infty}=471$ and $573 \mathrm{~m} / \mathrm{s}$, freestream Mach numbers were $M_{\infty}=1.81$ and 2.63, initial wake momentum thicknesses were $\vartheta=0.40 \mathrm{~mm}$ and $0.55 \mathrm{~mm}$, and the wake Reynolds numbers were $R e_{\vartheta}=3.1 \times 10^{4}$ and $2.1 \times 10^{4}$.

A shadowgraph system was used to image wave patterns in the Mach 2 and Mach 3 wakes. In addition, imaging of planar laser Mie scattering (PLMS) based on ethanol vapor seeding in air issuing from the slot jet was used to document the instantaneous two-dimensional structure and the visual width $\delta(x)$ of the wake. For these freestream temperature and flow conditions, the resulting ethanol fog droplet sizes are below $20 \mu \mathrm{m}$ (Ref. 27). A 532-nm laser sheet from a Nd:YAG laser with pulse duration $10 \mathrm{~ns}$ and energy of $80 \mathrm{~mJ} /$ pulse passed through the wake with $5 \mathrm{~mm}$ thickness. Mie scattering from the ethanol fog was imaged on a charge-coupled device (CCD) camera.

The sidewall static pressure distribution was monitored with a total of 16 sidewall pressure taps on the left and right sidewalls, as indicated in Fig. 1. From these, local values of the freestream Mach number $M_{\infty}(x)$ were obtained; note that $M_{\infty}$ varies with downstream position $x$ in a confined supersonic bluff-body wake due to the repeated reflections of the expansion and compression waves from the sidewalls. Static wall pressures were measured by taps on the front and back walls, each of which had three rows of 15 taps, one located on the test section centerline and two others located 0.4 in. on either side of the centerline. Pitot pressures were measured along the transverse direction in fine increments using a digital micrometer traverse at 21 downstream locations in the Mach 2 wake and at 16 downstream locations in the Mach 3 wake, using 0.025-in. $(600-\mu \mathrm{m})$ pitot probes. Using the measured wall static pressures,

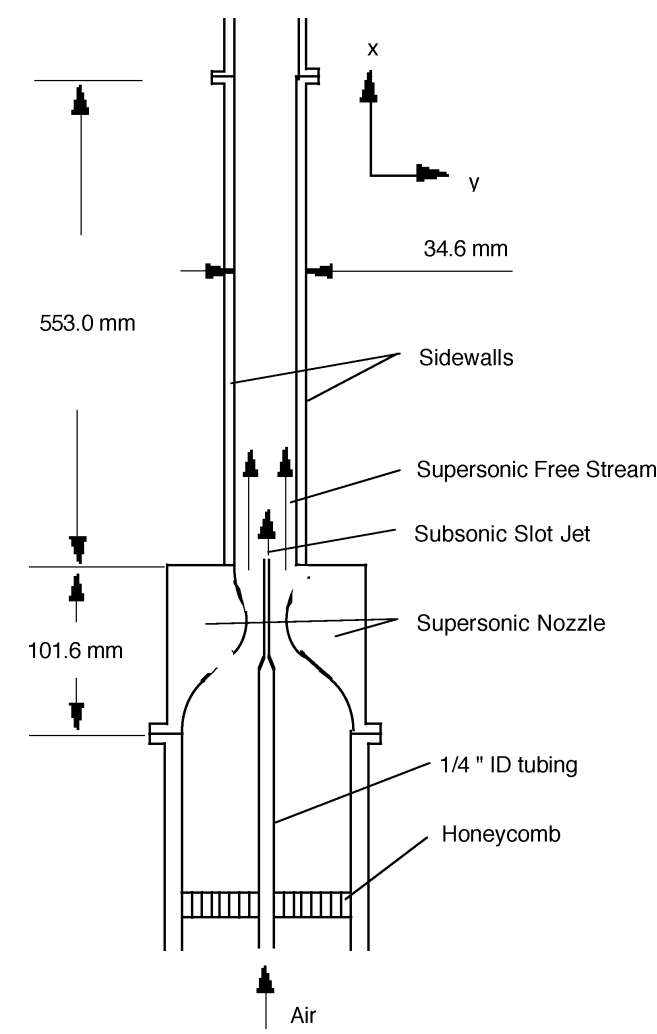

a) Front view

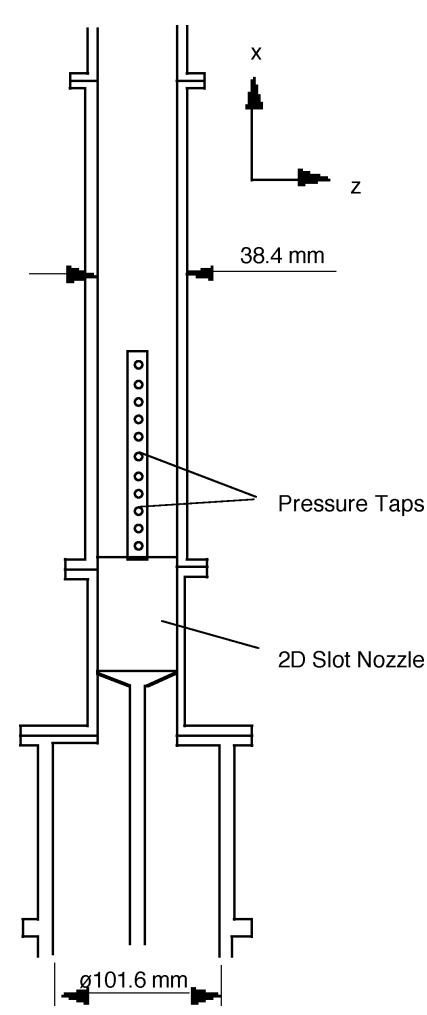

b) Side view

Fig. 1 Schematic of the supersonic nozzle, the test section, and the subsonic slot nozzle. 
the measured pitot pressure profiles were converted to Mach number profiles at each downstream location using the Rayleigh supersonic pitot formula and subsequently converted to velocity profiles.

\section{Planar Laser Mie Scattering and Shadowgraph Visualizations}

Figures 2 and 3 show typical examples of the instantaneous PLMS images for the Mach 2 and Mach 3 wakes, spanning from the nozzle exit well into the wake far field. In the Mach 2 wake, beginning at about $x=3 \mathrm{~cm}$, large-scale organized vortical structures with alternating signs of circulation can be seen to begin forming. By about $x=6 \mathrm{~cm}$, these structures have formed into a characteristic, quasiperiodic, vortex streetlike pattern that continues downstream. In the Mach 3 wake the Mie scattering intensity in the PLMS images is very weak up to about $x=4 \mathrm{~cm}$. Condensation of the ethanol vapor injected at the wake generator does not occur until the injected fluid mixes with the much colder supersonic freestream fluid. The volume fraction of cold freestream fluid in ethanol-containing fluid must be at least $20 \%$ for the ethanol vapor to begin condensing. ${ }^{9}$ This suggests that, upstream of $x=4 \mathrm{~cm}$ in the Mach 3 wake, the mixing between the slot-jet fluid and the freestream fluid has not reached this value, whereas in the Mach 2 wake sufficient mixing occurs almost
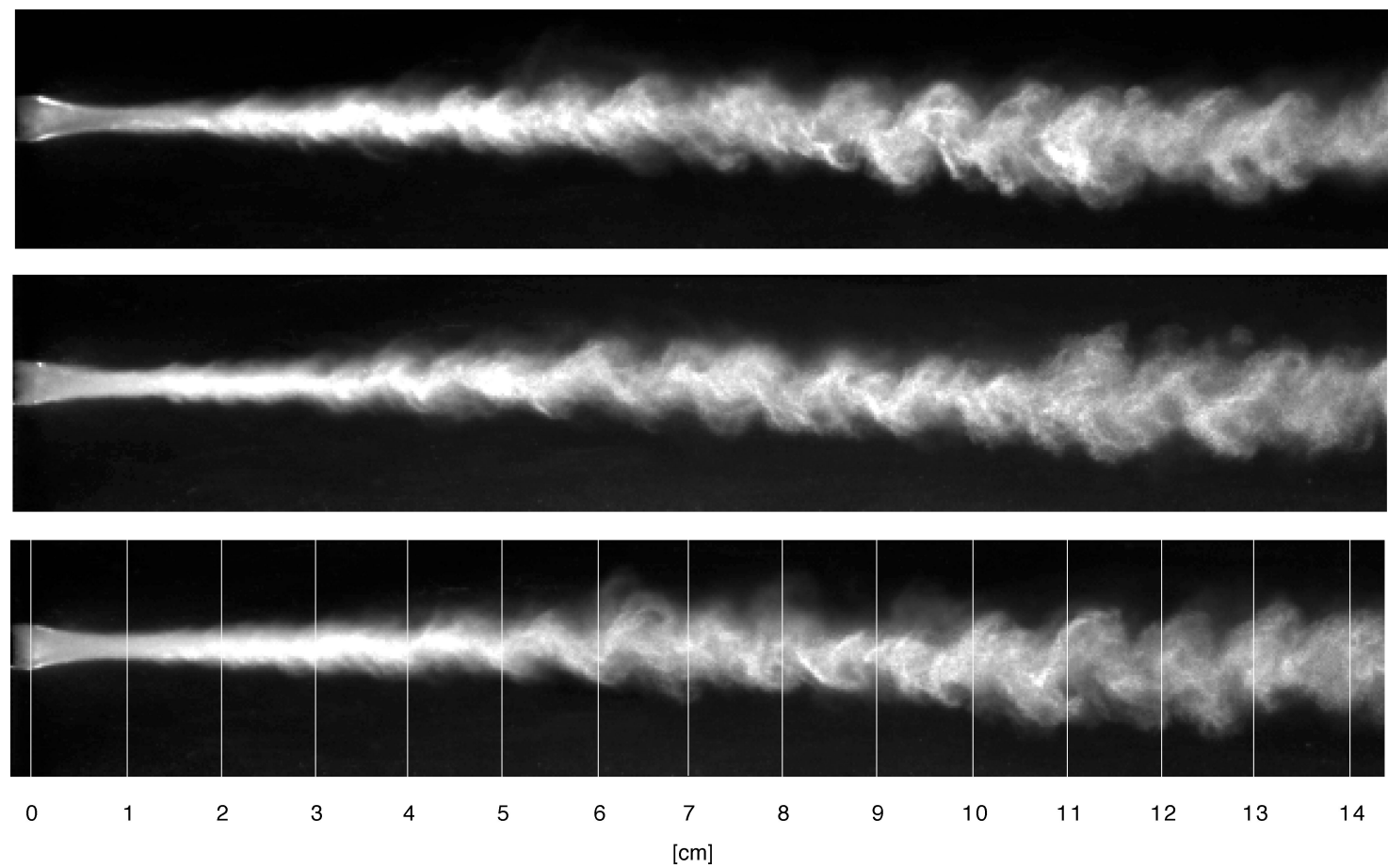

Fig. 2 Typical instantaneous PLMS images for the Mach 2 wake, showing development of characteristic vortex street-like large-scale structures in far-field region and the lack of any apparent structures immediately downstream of the base flow region, where the relative Mach number remains comparatively high.
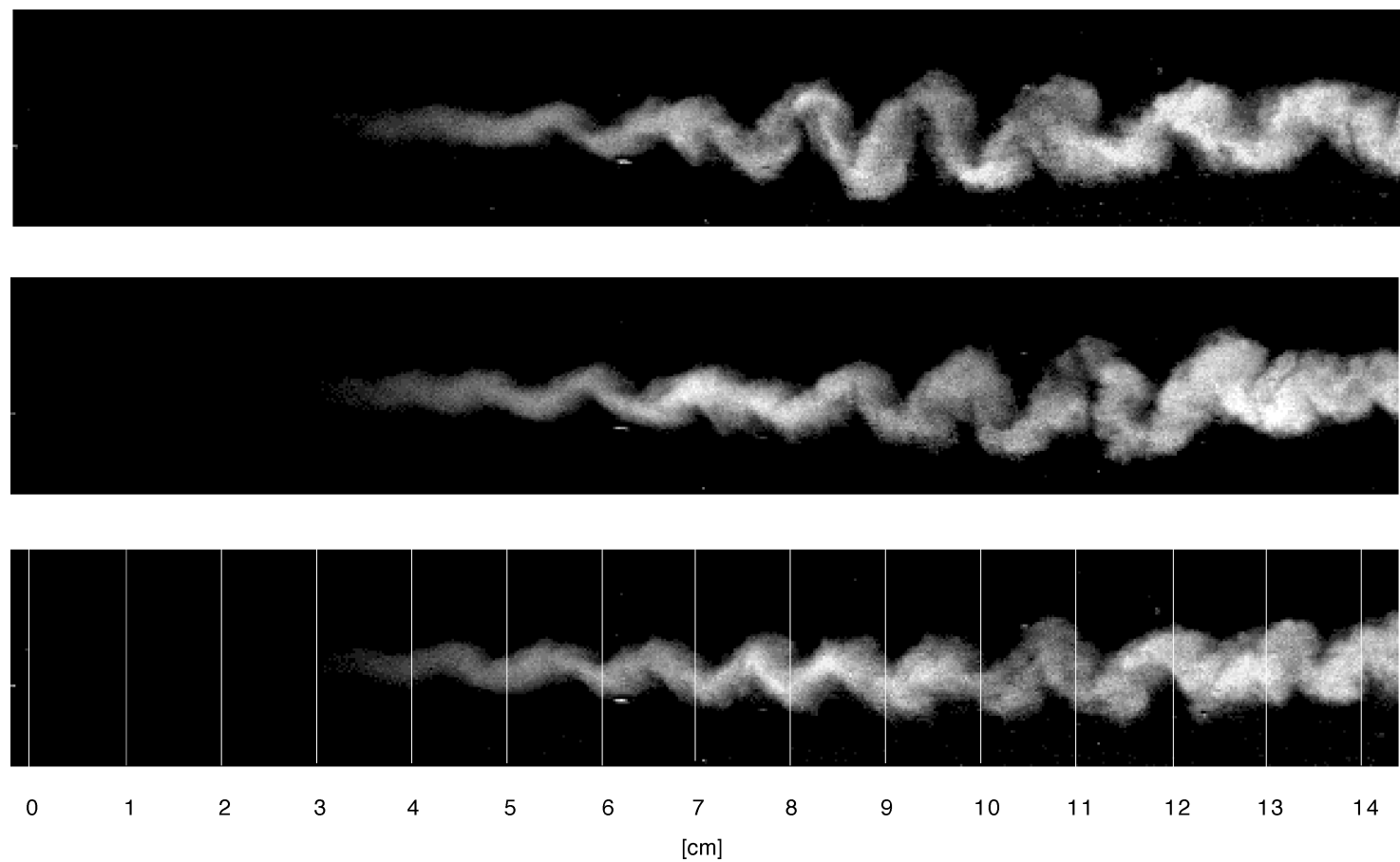

Fig. 3 Typical instantaneous PLMS images for the Mach 3 wake, showing dramatically different large-scale structure from those in Fig. 2 for the Mach 2 wake. Note that the PLMS signal is low for $x \leq 3.5 \mathrm{~cm}$ due to reduced mixing between slot jet and freestream where $M_{r}(x) \geq 0.4$. 
immediately beyond the slot-jet exit so that condensation can occur. Because the relative Mach number $M_{r}(x) \equiv M_{0}(x)-M_{\infty}(x)$, namely the local Mach number difference between the wake centerline and the free stream flow, is highest at these relatively small downstream locations in the Mach 3 wake, this suggests a reduction in the mixing rate in the supersonic wake with increasing relative Mach number. Further evidence of this will be seen in what follows.

Beginning at about $x=4 \mathrm{~cm}$ in the Mach 3 wake in Fig. 3, a distinct "zigzag" pattern appears that reflects the development of large-scale organized structures. The large-scale structures seen in Fig. 3 have a fundamentally different appearance than do the structures in the Mach 2 wake in Fig. 2. Over the range $6 \mathrm{~cm} \leq x \leq 10 \mathrm{~cm}$ the Mach 3 wake grows at a much faster rate than does the Mach 2 wake. Figure 3 shows that the increased wake width results principally from increased entrainment of freestream fluid into the wake, rather than from increased mixing between this freestream fluid and wake fluid. Beyond about $x=10 \mathrm{~cm}$ in Fig. 3, the Mach 3 wake appears to show a reduction in width due to detrainment of unmixed ambient fluid, instead of the continued growth that might normally be expected. The origins of this are examined in following sections.

Figures 4 and 5 show ensemble-averaged PLMS images, together with typical instantaneous shadowgraph images on which the mean PLMS images are superimposed. The growth in the visual thickness of the wake with increasing downstream distance can be clearly seen, as can the slight deflections of the wake by small asymmetries in the reflected expansion and compression wave patterns. At the higher Mach number, the angles of the expansion and recompression waves become shallower, and the reflected waves thus intersect the wake farther downstream than in the Mach 2 wake. Figure 5 also shows a dramatic increase in the growth rate of the wake, beginning near $x / \vartheta=120$, where $\vartheta$ is the initial wake momentum thickness, as was
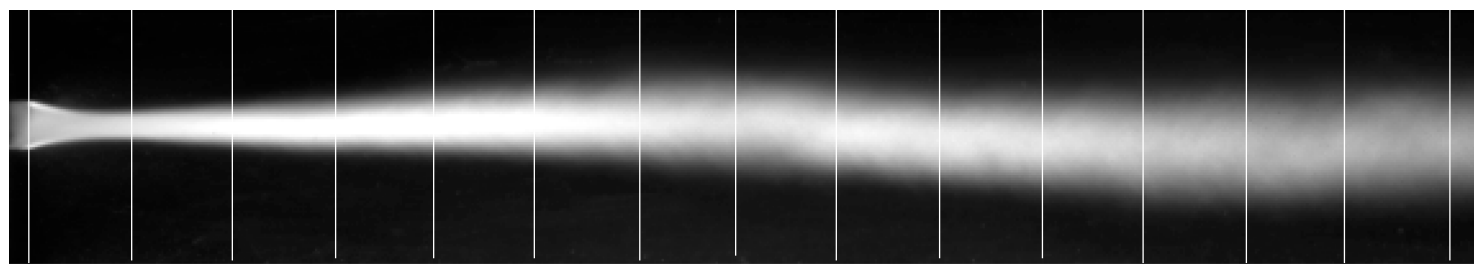

0

2

$3 \quad 4$

56

8

$9 \quad 10$

11

$12 \quad 13$

14

$[\mathrm{cm}]$

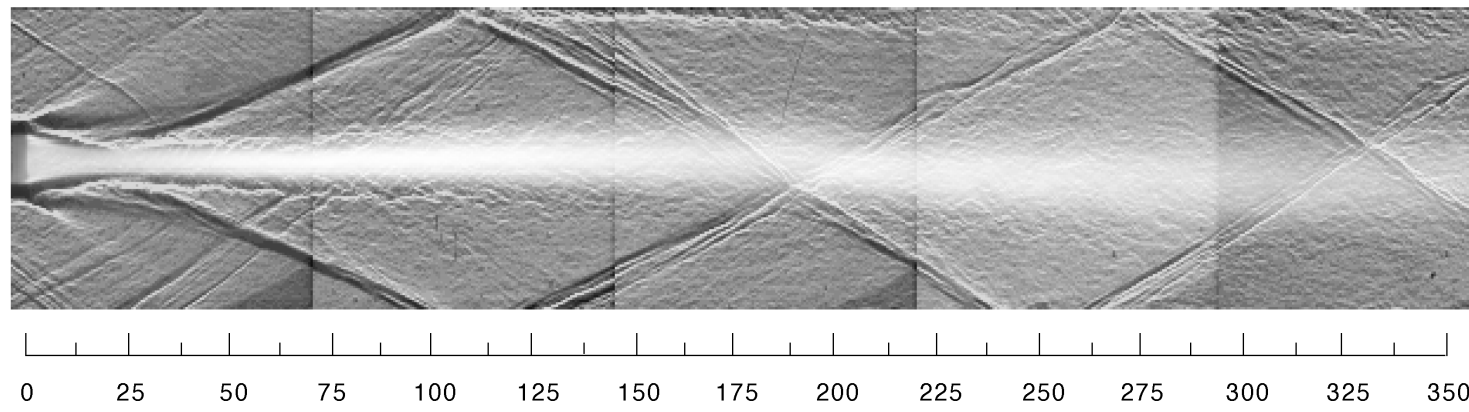

$[\mathrm{x} / \mathrm{\vartheta}]$

Fig. 4 Ensemble-averaged PLMS image for the Mach 2 wake (top) and instantaneous shadowgraph image showing wave structure (bottom). Scales show absolute downstream locations (centimeters) and corresponding relative downstream locations $(x / \vartheta)$ for the Mach 2 wake.

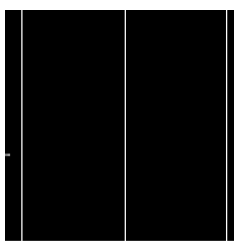

0

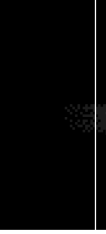

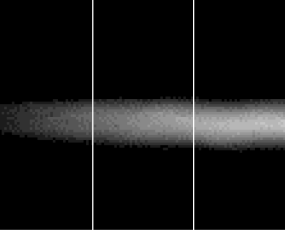

5

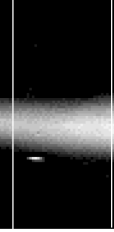

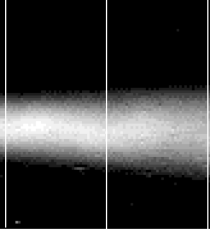

7

$[\mathrm{cm}]$

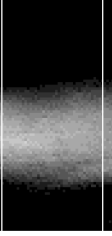

9

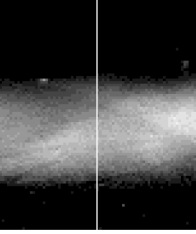

$10 \quad 11 \quad 12$

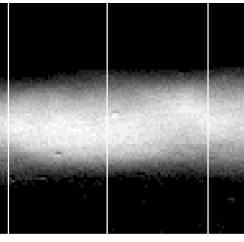

$2 \quad 13 \quad 14$

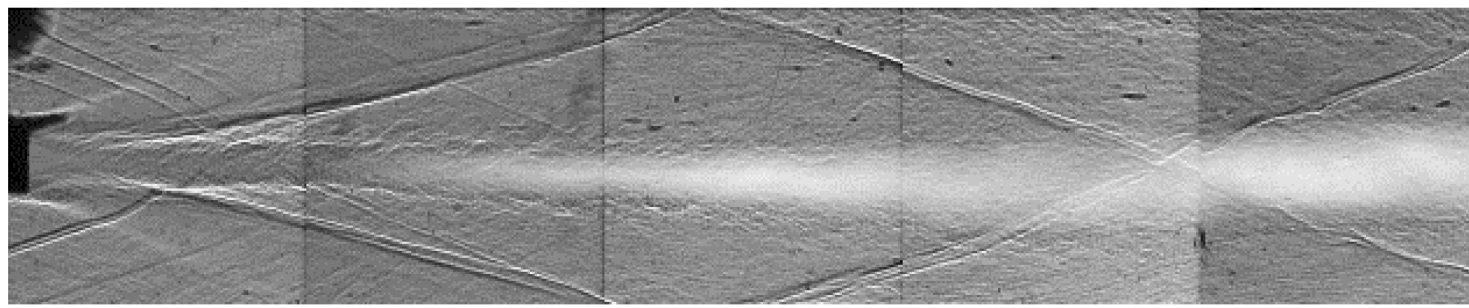

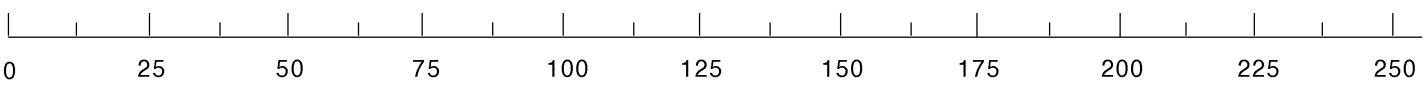

$[\mathrm{x} / \mathrm{\vartheta}]$

Fig. 5 Ensemble-averaged PLMS image for the Mach 3 wake (top) and instantaneous shadowgraph image (bottom); compare with Fig. 4. 


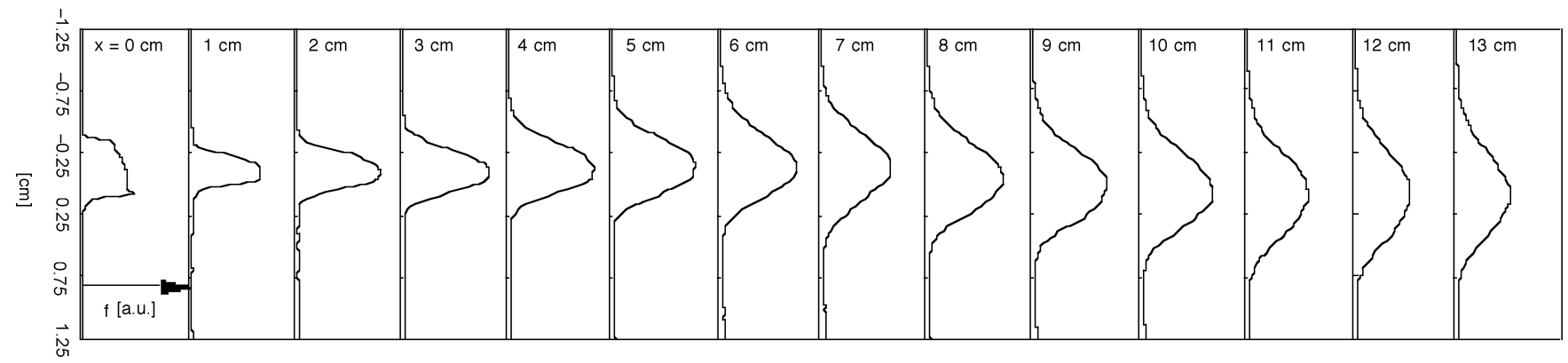

a) Mach 2 wake

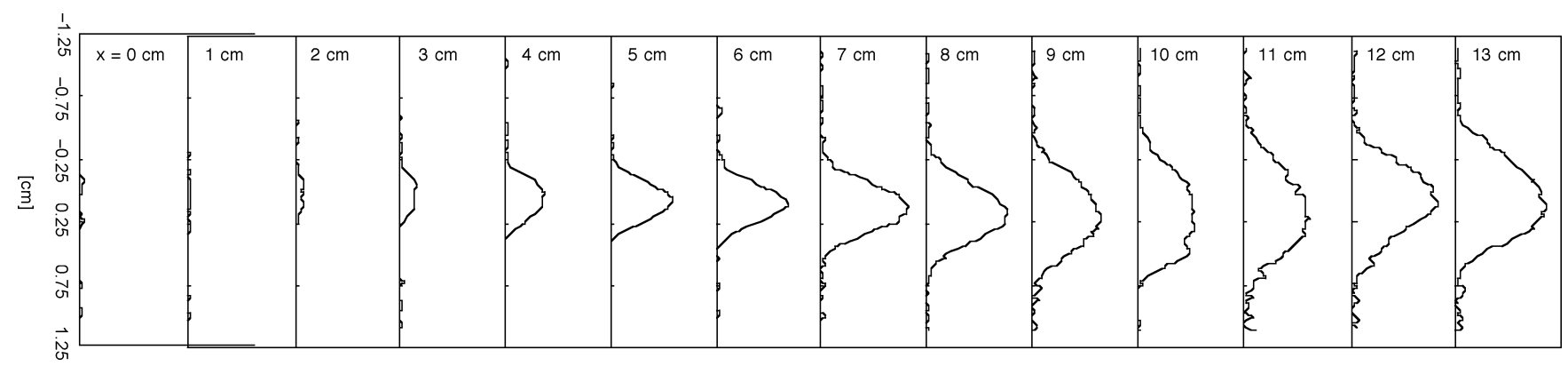

b) Mach 3 wake

Fig. 6 Ensemble-averaged PLMS intensity profiles at various downstream locations.

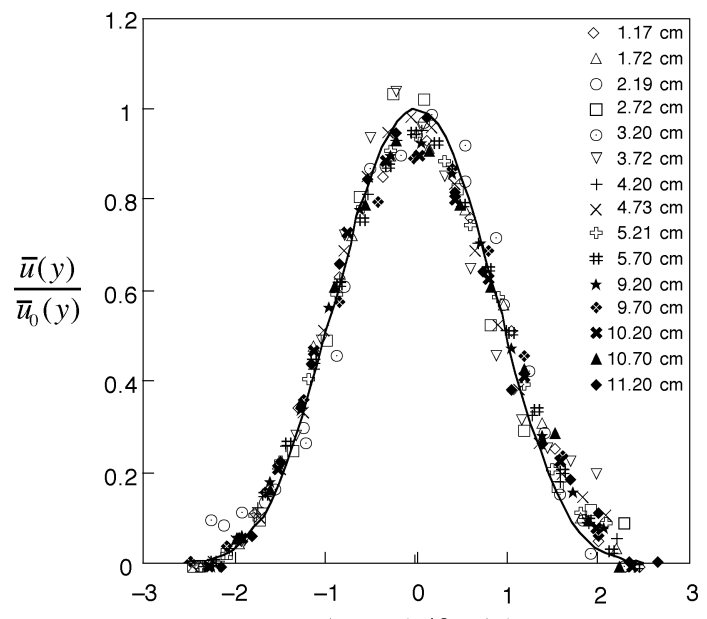

a) Mach 2 wake

$\left(y-y_{0}\right) / \delta_{1 / 2}(x)$

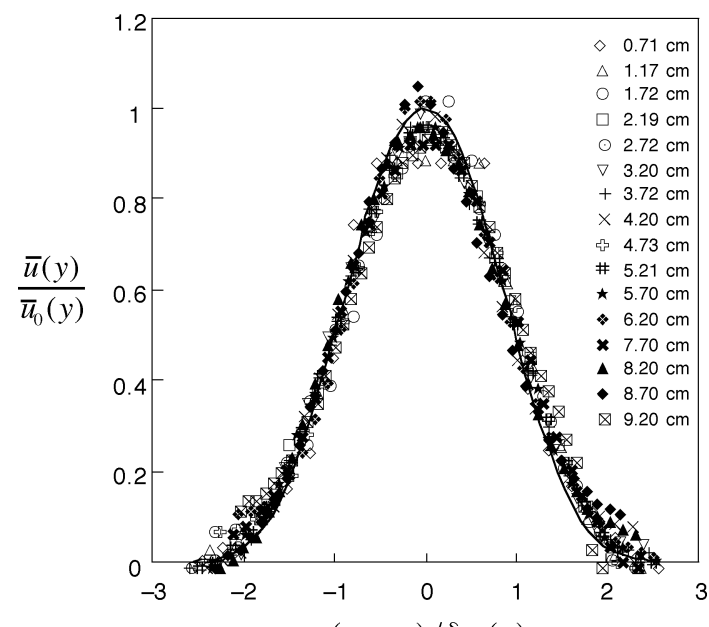

b) Mach 3 wake

$\left(y-y_{0}\right) / \delta_{1 / 2}(x)$

Fig. 7 Similarity of measured mean streamwise velocity profiles for Mach 2 and 3 wakes, showing good agreement of profile shapes at all downstream locations. Solid lines indicate the incompressible wake profile shape reported in Ref. 23.

suggested by the zigzag structure in Fig. 3, as well as the subsequent reduction in visual width of the wake.

Figure 6 shows lateral profiles of the mean PLMS intensity at several downstream locations in the Mach 2 and 3 wakes. To quantify the visual thickness of the wakes, a Gaussian fit was determined for each mean PLMS profile by matching the three lowest moments over the central portion of the flow to a Gaussian function. From these moments the local centerline position $y_{0}(x)$, the local maximum PLMS intensity, and the local visual width $\delta_{1 / 2}(x)$ of each profile were determined. Here $\delta_{1 / 2}(x)$ denotes the half-width at the halfmaximum point of the local intensity profile. The resulting $\delta_{1 / 2}(x)$ for each wake are examined below, together with analogous widths obtained from measured pitot pressure profiles in the following section.

\section{Velocity and Mach Number Results}

At each downstream position $x / \vartheta$ for the Mach 2 and 3 wakes, Fig. 7 shows the local mean velocity deficit profiles $u(y) \equiv U_{\infty}-U(y)$, where $U(y)$ are the mean streamwise velocity profiles obtained from the pitot probe measurements. Each profile is shown normalized by the local maximum value $u_{0}(x)$, and the lateral position $y$ from the maximum location $y_{0}$ is normalized by the local velocity half-width $\delta_{1 / 2}(x)$, where $u_{0}, y_{0}$, and $\delta_{1 / 2}$ were obtained from Gaussian fits to the mean velocity deficit profiles in the same manner as for the mean PLMS intensity profiles. In both cases the profiles are very nearly self-similar, except at the first two locations in the Mach 3 wake, where near-field effects lead to departures from self-similarity. The agreement among these profiles demonstrates that compressibility effects in supersonic wakes do not invalidate the fundamental self-similarity traditionally associated with incompressible turbulent shear flows, despite the complex wave interactions with the flow noted above and in Secs. V and VI.

Owing to the self-similarity in these mean profiles, compressibility effects on the mean flow properties can be determined from the local outer-variable scalings $\delta_{1 / 2}(x)$ and $u_{0}(x)$. These scalings in incompressible, planar, turbulent wakes are properly expressed in terms of the momentum thickness $\vartheta$, given by

$$
\vartheta \equiv\left(D-J_{0}\right) / \rho_{\infty} U_{\infty}^{2}
$$



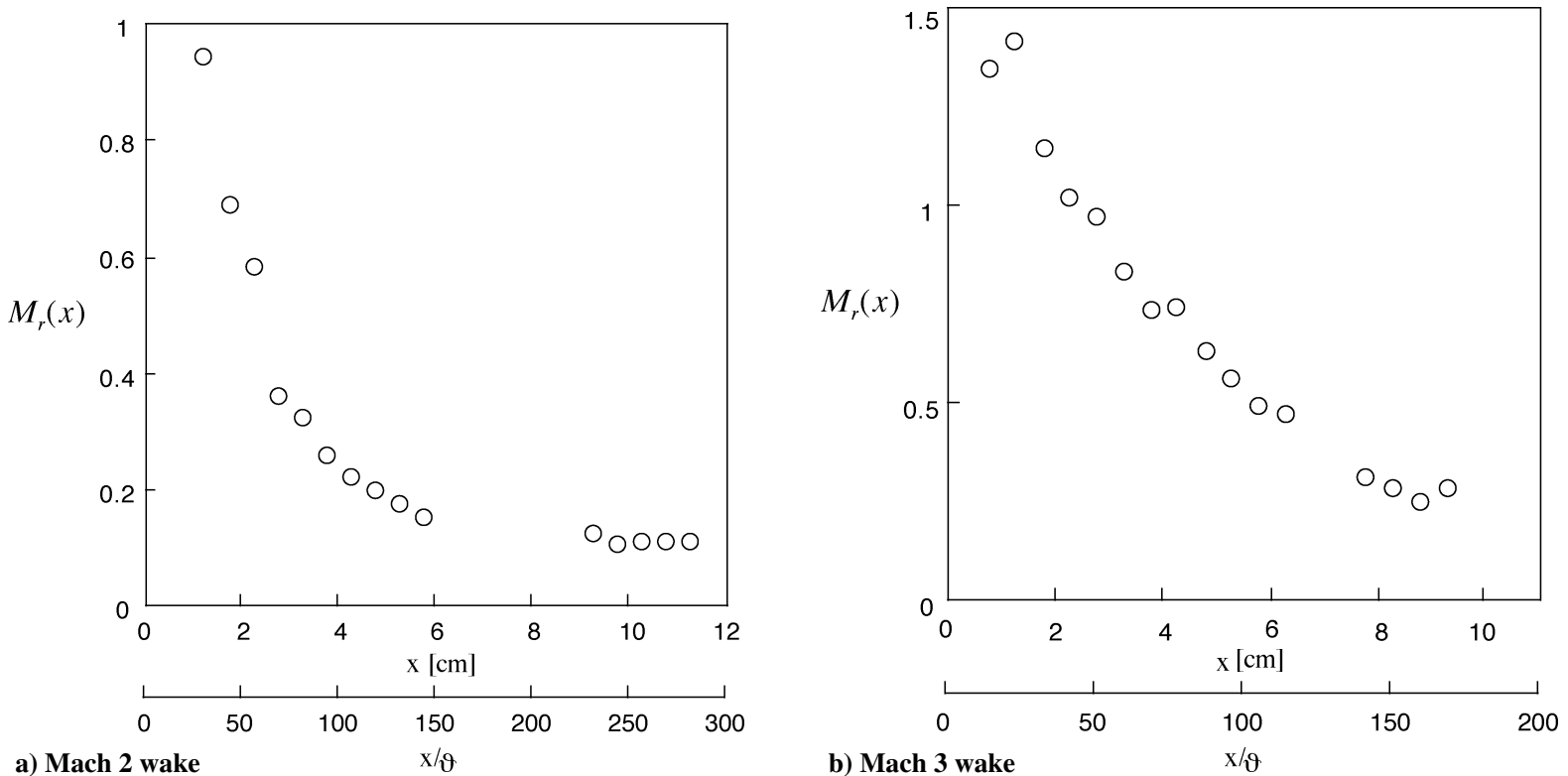

Fig. 8 Relative Mach number $M_{r}(x)$

which is invariant with downstream location $x$ in incompressible wakes. Indeed, the local momentum thickness $\vartheta(x)$ determined by integrating each of the velocity defect profiles in Fig. 7 was confirmed to be essentially invariant up to the $x$ location where the reflected expansion wave from the near-field recompression region first intersects the wake. The resulting values were found to be $\vartheta(x)=0.40 \pm 0.03 \mathrm{~mm}$ for the Mach 2 wake and $0.55 \pm 0.07 \mathrm{~mm}$ for the Mach 3 wake.

The relative Mach number $M_{r}(x)$ plays a role in supersonic wakes as an indicator of the local extent of compressibility to that of the convective Mach number $M_{c}$ in supersonic mixing layers. Here

$$
M_{r}(x)=u_{0}(x) / a_{\infty}
$$

where $a_{\infty}=\left(\gamma R T_{\infty}\right)^{\frac{1}{2}}$ and

$$
T_{0} / T_{\infty}=1+[(\gamma-1) / 2] M_{\infty}^{2}
$$

with $a_{\infty}$ and $T_{\infty}$ denoting the sound speed and temperature, respectively, in the freestream. Whereas in mixing layers $M_{c}$ does not vary with downstream location $x$, in wakes the velocity deficit $u_{0}(x)$ decreases with increasing $x$, and thus the relative Mach number $M_{r}(x)$ also decreases with $x$. As a result, supersonic turbulent wakes will evolve from comparatively large compressibility effects at relatively small values of the downstream location $x$, where $M_{r}(x)$ may be quite large, to an essentially incompressible wake flow at downstream locations sufficiently large for $M_{r}$ to have decreased to near-zero values. However, in confined supersonic wakes, even at $x$ locations where $M_{r}(x)$ has decreased to essentially incompressible values, local interactions of the wake with the reflected shock and expansion waves may still lead to compressibility effects, as will be seen in Secs. V and VI.

From the local measured $u_{0}(x)$ values in Fig. 7 and the corresponding freestream Mach number $M_{\infty}(x)$ obtained from the sidewall static pressures, Fig. 8 shows the resulting relative Mach number $M_{r}(x)$. In both the Mach 2 and 3 wakes, $M_{r}(x)$ decreases rapidly, and it is apparent that both wakes are at nearly incompressible local flow conditions over most of the downstream extent shown in Figs. 2-5. In supersonic turbulent mixing layers, reductions in the growth rate $\mathrm{d} \delta / \mathrm{d} x$ of at least $20 \%$ relative to the incompressible value are observed even at convective Mach numbers as low as 0.2. This suggests that comparable effects might be expected for $(x / \vartheta)<100$ in the Mach 2 wake and $(x / \vartheta)<150$ in the Mach 3 case, where $M_{r}(x)>0.2$.
Table 1 Scaling constants $c_{\delta}$ and $c_{u}$ from PLMS and velocity measurements in confined supersonic planar turbulent wakes, and corresponding scaling constants for unforced and forced

\begin{tabular}{|c|c|c|c|c|c|c|}
\hline \multirow[b]{2}{*}{ Quantity } & \multicolumn{2}{|c|}{$M_{\infty} \ll 1$} & \multicolumn{2}{|c|}{$M_{\infty} \approx 2$} & \multicolumn{2}{|c|}{$M_{\infty} \approx 3$} \\
\hline & Unforced & Forced & $\delta_{1 / 2}$ & $u_{0}$ & $\delta_{1 / 2}$ & $u_{0}$ \\
\hline$\left(x_{0} / \vartheta\right)_{\text {visual }}$ & - & - & -31 & 25 & -90 & 12 \\
\hline$\left(x_{0} / \vartheta\right)$ & -128 & -128 & 22 & 25 & 5 & 12 \\
\hline$c_{\delta}$ & $\begin{array}{l}0.27 \\
1.88\end{array}$ & $\begin{array}{c}0.35 \pm 0.03 \\
1.46 \pm 0.1\end{array}$ & 0.39 & $\overline{141}$ & 0.26 & 200 \\
\hline
\end{tabular}
incompressible planar turbulent wakes from Ref. 23

\section{Supersonic Wake Scaling Properties}

To look for such effects of the relative Mach number $M_{r}(x)$ in supersonic wakes, the measured local outer-variable scalings $\delta_{1 / 2}(x)$ and $u_{0}(x)$ are compared with corresponding results from incompressible wakes. In incompressible planar turbulent wakes, the local flow width scales as

$$
\delta_{\frac{1}{2}}(x) / \vartheta=c_{\delta}\left[\left(x-x_{0}\right) / \vartheta\right]^{\frac{1}{2}}
$$

and the centerline velocity deficit scales as

$$
u_{0}(x) / U_{\infty}=c_{\delta}\left[\left(x-x_{0}\right) / \vartheta\right]^{-\frac{1}{2}}
$$

The scaling constants $c_{\delta}$ and $c_{u}$ and the virtual origin $x_{0}$ depend on the wake generator. ${ }^{28,29}$ For incompressible planar turbulent bluffbody wakes, Ref. 28 reports

$$
\begin{gathered}
c_{\delta}=0.270 \\
c_{u}=1.88 \\
\left(x_{0} / \vartheta\right)=-128
\end{gathered}
$$

These constants are summarized in Table 1. For later reference, various other steady wake generators in Ref. 28 give the scaling constants as $c_{\delta}=0.29 \pm 0.03$ and $c_{u}=1.70 \pm 0.2$.

Based on the incompressible scaling in Eq. (4) in terms of the initial momentum thickness $\vartheta$, Fig. 9 shows $\left(\delta_{1 / 2} / \vartheta\right)^{2}$ vs $(x / \vartheta)$ for both the Mach 2 and 3 wakes. The circles denote the visual width $\delta_{1 / 2}(x)$ from the mean PLMS intensity profiles in Fig. 6 , and the squares denote the velocity width $\delta_{1 / 2}(x)$ from the mean velocity deficit profiles $u_{0}(x)$ in Fig. 7. If the present supersonic wakes follow the same $\frac{1}{2}$-power-law scalings as do incompressible wakes in terms 

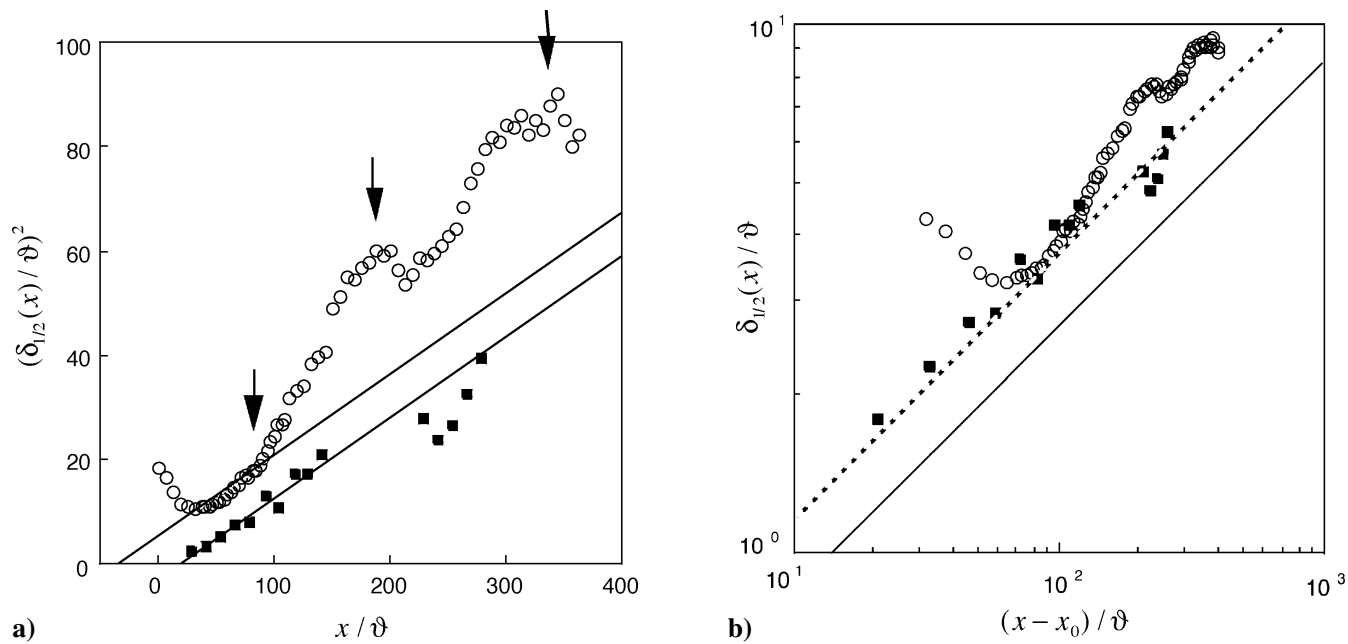

Fig. 9 Flow width scalings: a) measured flow width $\delta_{1 / 2}(x)$ for Mach 2 wake from mean PLMS intensity profiles (visual thickness) in Fig. 6a ( $\bigcirc$ ) and from mean velocity profiles (velocity thickness) in Fig. 7a (匹). Straight lines show $(\delta / \vartheta) \sim(x / \vartheta)^{1 / 2}$ scalings and allow virtual origins $x_{0}$ and scaling constants $c_{\delta}$ to be determined. Two peaks in the visual thickness indicated by black arrows near $(x / \vartheta)=180$ and 330 coincide with locations of recompression wave intersections in Fig. 4. Arrow near $(x / \vartheta)=90$ indicates location where leading characteristic of expansion wave intersects wake; b) flow widths $\delta_{1 / 2}(x)$ in $\log$-log form, with lines indicating $\frac{1}{2}$-power-law scaling for near-field scaling constant $c_{\delta}$ from forced incompressible wakes (-- ) and unforced incompressible wakes (-) from Ref. 23.

of the initial $\vartheta$, then the symbols in Fig. 9 should fall on straight lines, with the slopes being related to the scaling constant $c_{\delta}$, and the virtual origin $\left(x_{0} / \vartheta\right)$ being identifiable by the $x$ intercepts. The results for the Mach 2 and 3 wakes are discussed separately next.

\section{A. Mach 2 Wake Scaling}

The first few circles in Fig. 9a, giving the visual width $\delta_{1 / 2}(x)$ for the Mach 2 wake, are within the near-field base-flow region and thus show $\delta_{1 / 2}(x)$ decreasing with increasing $x$ due to the narrowing effect of the expansion and recompression process. Beyond the recompression zone, the wake widths $\delta_{1 / 2}(x)$ for the next 15 points increase in accordance with the $\frac{1}{2}$-power-law scaling evident from the straight line. Because these points are upstream of the first intersection of the wake by the reflected expansion wave, the momentum thickness remains constant at the initial value $\vartheta$. The virtual origin implied by the $x$ intercept based on these points gives

$$
\left(x_{0} / \vartheta\right)_{\mathrm{vis}}=-31
$$

and the slope gives the scaling constant as

$$
c_{\delta}=0.39
$$

The downstream shift in the virtual origin in Eq. (9) relative to the incompressible value in Eq. (8) is consistent with the results in Ref. 29. However, it is remarkable that $c_{\delta}$ in Eq. (10) is substantially higher than the incompressible value in Eq. (6), because experience from supersonic mixing layers might suggest a reduction in $c_{\delta}$ due to compressibility. Whereas the value in Eq. (10) is significantly higher than that found for any steady-wake generator in Ref. 28, the same study found that periodic forcing introduced at the wake generator can dramatically increase the scaling constant $c_{\delta}$ over the value obtained from the same generator without forcing (see Table 2 in Ref. 28). Such forced wakes typically produced values in the range of

$$
c_{\delta}=0.35 \pm 0.03
$$

depending on the details of the forcing. This compares well with the present value in Eq. (10), and it will be seen later that there is strong evidence that confined supersonic bluff-body wakes are subject to wave-induced periodic forcing from interactions of the reflected expansion waves with the organized large-scale vortical structures of the flow. The remainder of the circles in Fig. 9a, which depart from the straight line for the $\frac{1}{2}$-power-law scaling, are related to effects of such forcing and will be discussed later.
The squares in Fig. 9a give the velocity width $\delta_{1 / 2}(x)$ obtained from the mean velocity profiles in Fig. 7. The first 10 of these points, for which the momentum thickness remains constant, follow the same $\frac{1}{2}$-power-law scaling indicated by the straight-line fit through them, and give the same value for the scaling constant as in Eq. (10), namely $c_{\delta}=0.39$. Only the virtual origin is different for the velocity width, giving

$$
\left(x_{0} / \vartheta\right)=22
$$

The fact that the virtual origin for the visual thickness in Eq. (9) is more negative than that for the velocity width in Eq. (12) is consistent with the common observation that the scalar (visual) width of turbulent shear flows is greater than the velocity profile width.

Based on the corresponding $\frac{1}{2}$-power-law scaling in Eq. (5) for the mean velocity deficit in incompressible turbulent wakes, Fig. 10a shows $\left(u_{0} / U_{\infty}\right)^{-2}$ vs $(x / \vartheta)$ for the Mach 2 wake. The centerline velocity deficit scaling shows similar features as did the independently measured flow width scalings in Fig. 9a. Over roughly the same $x$ range where $\delta_{1 / 2}(x)$ followed the incompressible scaling, $u_{0}(x)$ in Fig. 11a also follows the $-\frac{1}{2}$-power-law incompressible wake scaling. The departure from this straight-line scaling begins at the same $x$ location, indicated by the first arrow, at which $\delta_{1 / 2}(x)$ departed from its scaling. Moreover, the virtual origin in Fig. 10a is

$$
\left(x_{0} / \vartheta\right)=25
$$

in good agreement with the value in (12) obtained from the velocity profile widths given by the solid symbols in Fig. 9a. The slope of the line in Fig. 10a gives the scaling constant as

$$
c_{u}=1.41
$$

In agreement with the results in Fig. 9, this is lower than the value in Eq. (7) found in Ref. 28 for unforced incompressible wakes and is close to the value

$$
c_{u}=1.46 \pm 0.1
$$

found in Ref. 28 for forced incompressible wakes. This can be seen in $\log -\log$ form in Fig. 10b, where the dashed and solid lines correspond respectively to the $-\frac{1}{2}$-power-law scalings with the scaling constants $c_{u}$ for forced and unforced incompressible wakes. Based on these independent measurements of the flow width scaling $\delta_{1 / 2}(x)$ and the centerline velocity deficit scaling $u_{0}(x)$ in the Mach 2 wake, 

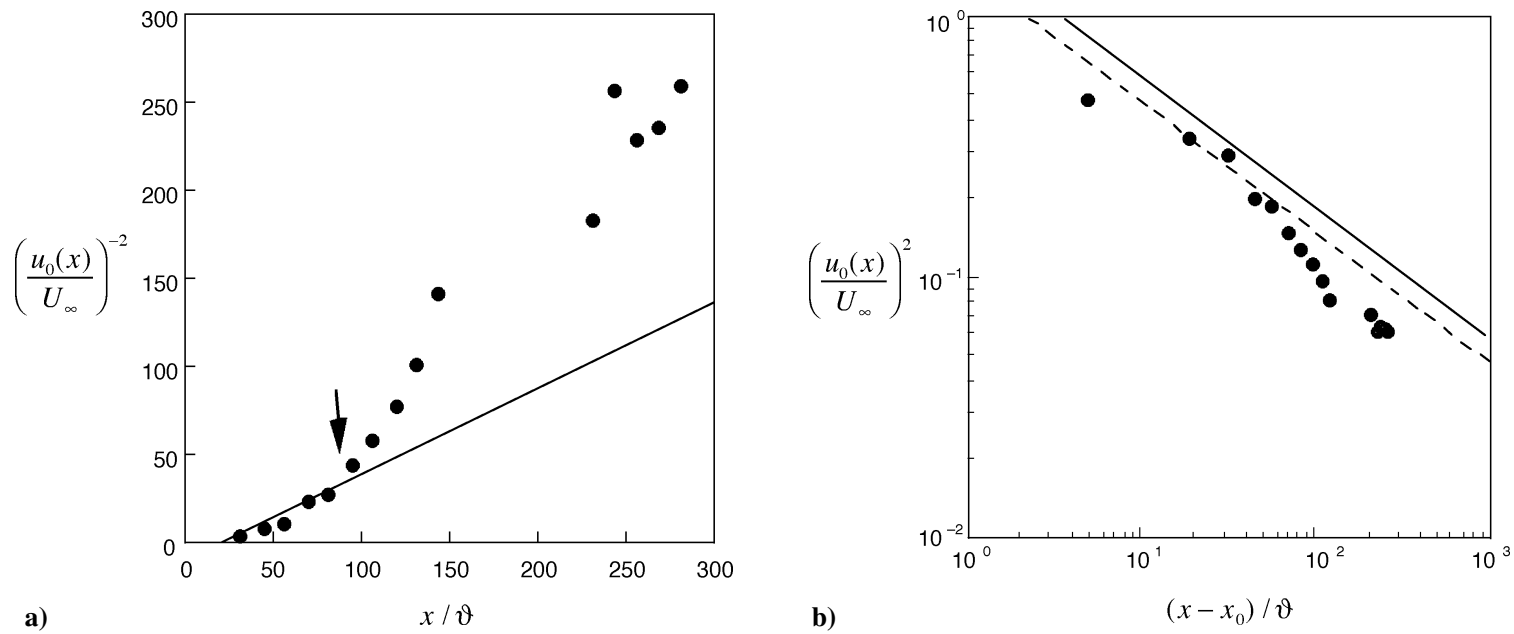

Fig. 10 Centerline velocity deficit scalings: a) wake centerline velocity deficit $u_{0}(x)$ for Mach 2 wake from velocity profiles in Fig. 7 a. Straight lines indicate $\left(u_{0} / U_{\infty}\right) \sim(x / \vartheta)^{-1 / 2}$ scalings, allowing virtual origin $x_{0}$ and scaling constant $c_{u}$ to be determined. Arrow indicates where leading characteristic of expansion wave intersects the wake; b) wake centerline velocity deficit $u_{0}(x)$ in $\log$-log form, with lines indicating $-\frac{1}{2}$-power-law scaling for values of the scaling constant $c_{u}$ from forced incompressible wakes (---) and unforced incompressible wakes ( $\longrightarrow$ ) from Ref. 23.
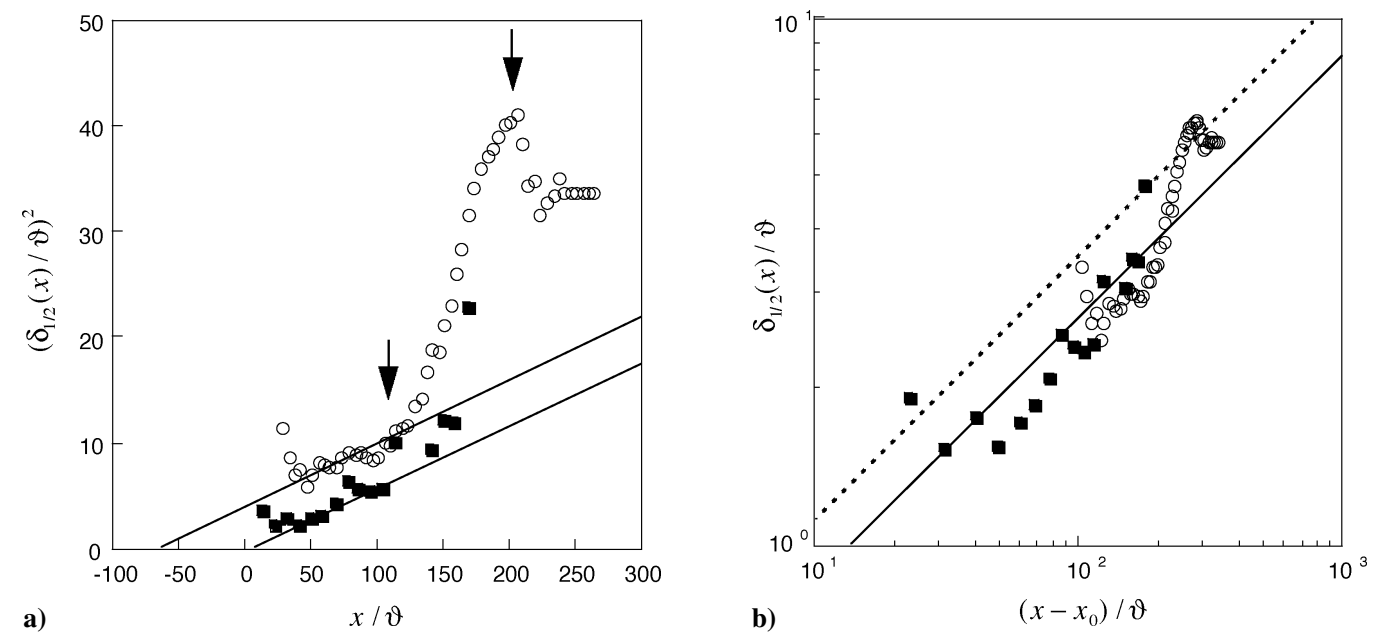

Fig. 11 Flow width scalings: a) measured flow width $\delta_{1 / 2}(x)$ for Mach 3 wake from mean PLMS intensity profiles (visual thickness) in Fig. 6b (O) and from mean velocity profiles (velocity thickness) in Fig. $7 \mathrm{~b}(\square)$. Straight lines show $(\delta / \vartheta) \sim(x / \vartheta)^{1 / 2}$ scalings and give virtual origins $x_{0}$ and scaling constants $c_{\delta}$. Peak in the visual thickness indicated by arrow near $(x / \vartheta)=190$ coincides with location of recompression wave intersection in Fig. 5 . Arrow near $(x / \vartheta)=120$ indicates where leading characteristic of expansion wave intersects wake; b) flow widths $\delta_{1 / 2}(x)$ in log-log form, with lines indicating $\frac{1}{2}$-power-law scaling for near-field scaling constant $c_{\delta}$ from forced incompressible wakes (---) and unforced incompressible wakes $(-)$ from Ref. 23 .

it is apparent that, upstream of the location where the reflected expansion wave in Fig. 4 first intersects the wake, the flow follows the same power-law scalings and scaling constants as are found in Ref. 28 for forced incompressible wakes.

For $(x / \vartheta) \geq 90, \delta_{1 / 2}(x)$ in Fig. 9a no longer follows the $\frac{1}{2}$-powerlaw scaling of incompressible wakes, even though at these large $x$ locations the relative Mach number $M_{r}(x)$ in Fig. 8 has decreased to essentially incompressible values. The origin of the departures from the $\frac{1}{2}$-power-law scaling can be understood by noting that the symbols in Figs. 9a and 10a show two peaks centered roughly at $(x / \vartheta)=190$ and 330, as indicated by the arrows, corresponding respectively to $x=7.6$ and $13.0 \mathrm{~cm}$. These positions coincide with the locations in Fig. 4 where the reflected recompression shocks intersect the Mach 2 wake. In addition, the leading Mach wave of the reflected expansion wave in Fig. 4 first intersects the wake at $(x / \vartheta)=90$, indicated by the first arrow in Fig. 9a, corresponding to $x=3.6 \mathrm{~cm}$, which is precisely where the circles in Fig. 9a begin to depart from the $\frac{1}{2}$-power-law scaling. These observations suggest that the deviations from the $\frac{1}{2}$-power-law scaling in Fig. 9a are connected to these wave interactions with the flow. Moreover, in Fig. $9 \mathrm{~b}$ the same data for the visual and velocity widths $\delta_{1 / 2}(x)$ are shown in log-log form, with the $\frac{1}{2}$-power-law scalings given by the dashed and solid lines corresponding to the scaling constants $c_{\delta}$ from Ref. 28 for forced and unforced wakes.

The change in density within the wake as a result of the wave intersection would be expected to produce changes in the local momentum thickness, and thus at least part of the departures from the incompressibile scalings in Figs. 9a and 9b may be attributable to simple density changes. However, the fact that, upstream of the first wave intersection with the flow, the results for the Mach 2 wake follow the forced wake scaling constants from Ref. 28 suggests that there may be a further mechanism contributing to the local departures from the $(\delta / \vartheta) \sim(x / \vartheta)^{1 / 2}$ scaling, namely a local forcing of the wake due to the reflected wave interactions. This will be considered further after corresponding results from the Mach 3 wake have been discussed.

\section{B. Mach 3 Wake Scaling}

Figure 11 presents results for the Mach 3 wake analogous to those given in Fig. 9 for the Mach 2 case. In the Mach 3 wake, the $x$ location at which the reflected expansion wave first intersects the wake occurs further downstream than in the Mach 2 case. Figure 11a shows $\left(\delta_{1 / 2} / \vartheta\right)^{2}$ vs $(x / \vartheta)$, with circles denoting the visual width $\delta_{1 / 2}(x)$ from mean PLMS intensity profiles in Fig. $6 \mathrm{~b}$, and squares 

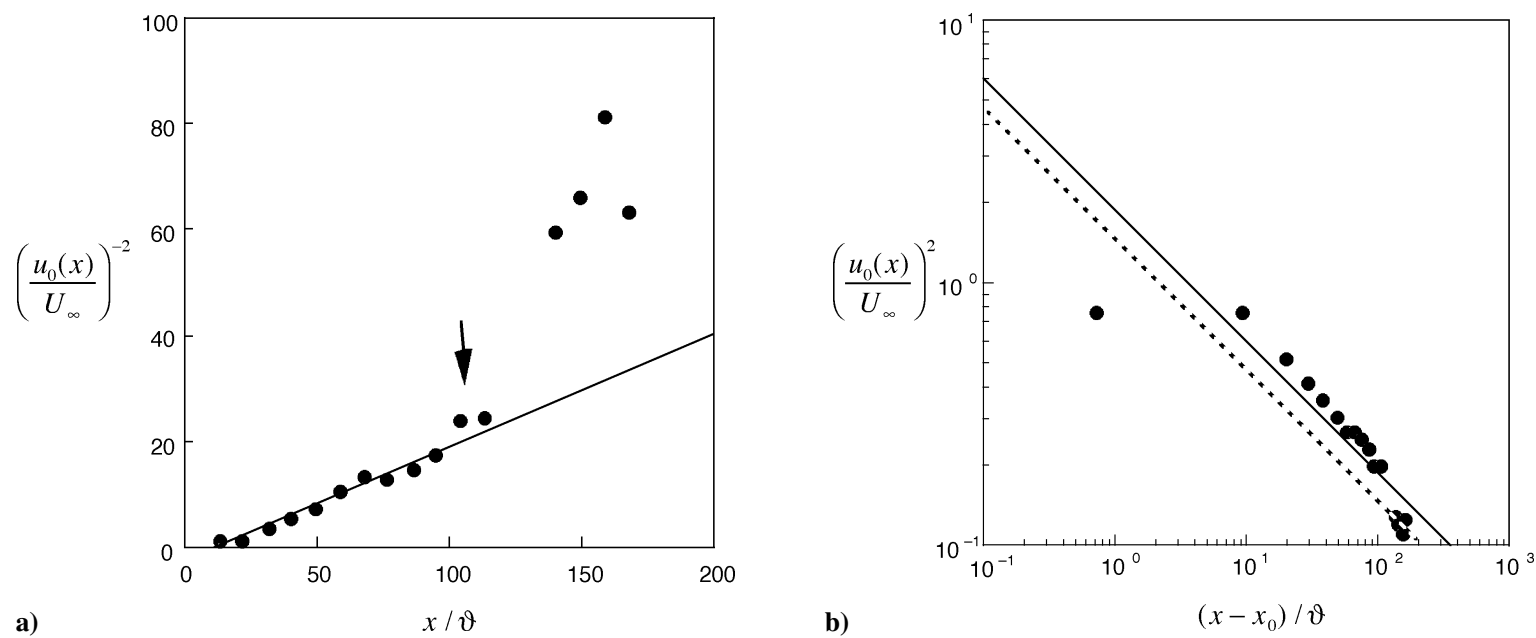

Fig. 12 Centerline velocity deficit scalings: a) wake centerline velocity deficit $u_{0}(x)$ for Mach 3 wake from velocity profiles in Fig. $7 \mathrm{~b}$. Straight lines indicate $\left(u_{0} / U_{\infty}\right) \sim(x / \vartheta)^{-1 / 2}$ scalings, allowing virtual origin $x_{0}$ and scaling constant $c_{u}$ to be determined. Arrow indicates where leading characteristic of expansion wave intersects the wake; b) wake centerline velocity deficit $u_{0}(x)$ in $\log$-log form, with lines indicating - $\frac{1}{2}$-power-law scaling for values of the scaling constant $c_{u}$ from forced incompressible wakes (-- ) and unforced incompressible wakes (-

denoting the velocity width $\delta_{1 / 2}(x)$ from $u_{0}(x)$ in Fig. $7 \mathrm{~b}$. Except for the first few points in the near-field recompression region, both profiles follow the $\frac{1}{2}$-power-law scaling of incompressible wakes indicated by the straight lines, up to about $(x / \vartheta) \approx 120$. Recall that the momentum thickness remains constant at its initial value $\vartheta$ up to this point. The virtual origins for the visual and velocity widths implied by the intercepts of these lines are

$$
\begin{gathered}
\left(x_{0} / \vartheta\right)_{\text {vis }}=-90 \\
\left(x_{0} / \vartheta\right)=5
\end{gathered}
$$

which are again shifted downstream from the value in Eq. (8) for incompressible wakes. ${ }^{28,29}$ The scaling constant $c_{\delta}$ implied by both straight-line slopes is again the same for both the visual and velocity widths, and corresponds to

$$
c_{\delta}=0.26
$$

Note that this is much lower than the value in Eq. (10) from the Mach 2 wake and is comparable to $c_{\delta}$ in Eq. (6), found in Ref. 28 for unforced incompressible wakes.

Figure 12 shows $\left(u_{0} / U_{\infty}\right)^{-2}$ vs $(x / \vartheta)$ for the Mach 3 wake, where it is evident that the data follow $-\frac{1}{2}$-power-law scaling up to the point where the expansion wave first intersects the wake, indicated by the arrow. The virtual origin was found to be

$$
\left(x_{0} / \vartheta\right)=12
$$

This is in reasonable agreement with the value in Eq. (17) for velocity profile widths and with the result in Ref. 29. The scaling constant from the slope in Fig. 12a is

$$
c_{u}=2.00
$$

and this is also comparable to the value in Eq. (7) from Ref. 28 for unforced, incompressible, bluff-body wakes. This is verified by the $\log -\log$ form in Fig. 12b, where the present data are shown together with solid and dashed lines that, respectively, give the unforced and forced incompressible scalings from Ref. 28. Thus unlike the Mach 2 wake, the Mach 3 wake follows the same power-law scalings and scaling constants, for both $\delta_{1 / 2}(x)$ and $u_{0}(x)$, as found in unforced incompressible wakes up to the $x$ location at which the reflected expansion wave first intersects the wake.

Beyond $(x / \vartheta) \approx 120$, the data in Fig. 11a depart from the straight line, as was seen in Fig. 9a, despite the fact that the relative Mach number $M_{r}(x)$ in Fig. 8 has decreased to nearly incompressible values. In this case, the departure shows only one peak over the range of downstream distances considered, now centered roughly at $(x / \vartheta)=190$, as indicated by the arrow, which corresponds to $x=10.5 \mathrm{~cm}$. This coincides with the location in Fig. 5 where the reflected recompression shocks intersect the Mach 3 wake. Moreover, the point where the leading Mach wave of the reflected expansion wave in Fig. 5 first intersects the wake is at $(x / \vartheta) \approx 120$, indicated by the first arrow in Fig. $11 \mathrm{a}$, which corresponds to $x=6.6 \mathrm{~cm}$. This again is precisely where the data in Fig. 11a begin to depart from the $\frac{1}{2}$-power-law scaling.

As in the Mach 2 wake, at least some of the departures from the incompressible scaling are due to density changes produced by the reflected waves, which create changes in the local momentum thickness. However, as was suggested by the results from the Mach 2 wake, these further observations in the Mach 3 wake give additional indications that some of the departures from the $\frac{1}{2}$-power-law scalings in Figs. 11 and 12 may be directly connected with a dynamical effect of the wave interactions with the wake flow. In particular, the Mach 2 wake, which allowed upstream propagation of disturbances from the reflected-wave intersection point, was seen to follow the forced wake scaling constants up to the first intersection point. In contrast to this, the Mach 3 wake, for which no upstream propagation was possible for any disturbances introduced by the interaction of the reflected wave with the wake, was seen to follow the unforced wake scaling constants up to the first intersection point. This indicates that the reflected wave interaction with the wake introduces some type of local forcing in the wake. Forcing is known to have a significant effect on the growth rate $\mathrm{d} \delta / \mathrm{d} x$ of incompressible wakes, suggesting the possibility of changes in the growth rate of supersonic wakes as a result of such wave-induced local forcing. This is examined in the Sec. VI.

\section{Wave-Induced Forcing Effects}

The fact that the Mach 2 wake in Fig. 9b follows the forced scaling constant given by the dashed line, whereas the Mach 3 wake in Fig. 11b follows the unforced scaling constant given by the solid line, provides important information on the mechanism by which wave interaction with the wake causes the departures seen in Figs. 912 from the nominal wake power-law scalings. The intersection of the reflected expansion wave with the wake in confined supersonic bluff-body wakes results in forcing of the flow induced locally by interaction between the large-scale vortical structures in the wake far field and the pressure field established by the reflected expansion wave as it intersects with the flow. At the wave intersection point, the forcing frequency will be automatically matched to the characteristic frequency $u_{0}(x) / \delta(x)$ of the large-scale vortical structures in the wake, irrespective of the downstream location $x$ at which the intersection occurs, because it is the passage of the vortices through the stationary wave that is central to the forcing. Consequently, the local large-scale vortices will automatically "lock on" to the resulting 

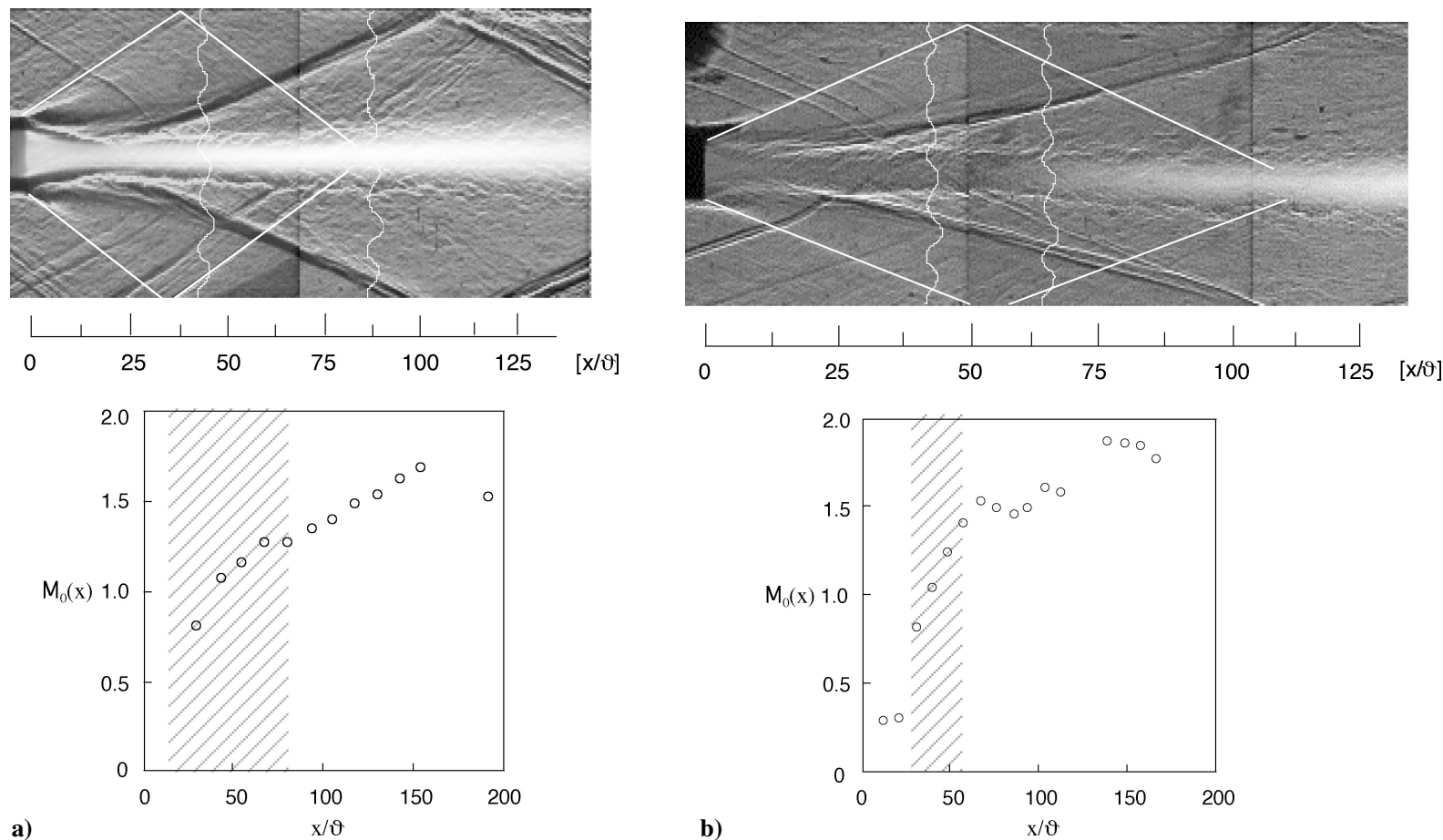

Fig. 13 Feedback mechanism leading to change from forced scaling constant $c_{\delta}$ in Mach 2 wake to unforced scaling constant in Mach 3 wake, showing shadowgraph image for a) Mach 2 and b) Mach 3 wakes. White lines indicate leading characteristic of expansion wave and approximate region where centerline Mach number changes from subsonic and supersonic. If expansion wave intersects wake where the local average centerline Mach number $M_{0}(x)$ is sufficiently close to subsonic, as shown in the bottom figures (shaded), then instantaneous subsonic path exists for local wave-induced forcing to propagate upstream to bluff body, leading to forced-wake flow in the Mach 2 wake and unforced-wake flow in the Mach 3 wake.

forcing frequency, in contrast to the externally introduced periodic disturbances that are typically used to force the growth rate of turbulent shear flows ${ }^{30-33}$ for which the frequency must be tuned to the local characteristic frequency of the large-scale structures. This selfexcited forcing mechanism in the confined supersonic wake causes departures of $\delta(x)$ and $u_{0}(x)$ from their power-law scalings past the point where the reflected expansion wave first intersects the wake.

The effect of this local forcing is to produce a rapid downstream increase in the growth rate of the wake, consistent with previous studies $^{25}$ of forced incompressible wakes, leading to a local departure from the $\frac{1}{2}$-power-law scaling beginning immediately downstream of the point where the expansion wave intersects the wake. Furthermore, the ability of this locally induced forcing to propagate upstream, and thereby affect the growth rate of the entire wake, depends on the existence of a subsonic path near the flow centerline. The presence of such a path depends on the centerline Mach number $M_{0}(x)=M_{\infty}(x)-M_{r}(x)$. As shown in Fig. 13, in the Mach 2 wake $M_{0}(x)$ is sufficiently low at the location $(x / \vartheta) \approx 90$ where the reflected expansion wave first intersects the wake so that such an instantaneous subsonic upstream path will extend to the wake generator. In the Mach 3 wake, however, two factors prevent such a subsonic path. First, the centerline Mach numbers $M_{0}(x)$ are inherently higher throughout the wake. Additionally, due to the shallower wave angles, the downstream location at which the reflected expansion wave first interacts with the wake is moved much farther downstream, where the centerline Mach number $M_{0}(x)$ is supersonic. As a result, no subsonic upstream path exists along which the local forcing produced by the wave interaction with the wake can propagate upstream. Consequently, upstream of the first expansion wave interaction the Mach 3 wake is unforced and thus grows with the unforced scaling constant $c_{\delta}$ in (18), whereas in the Mach 2 wake the forcing propagates upstream and the wake in that case grows with the forced scaling constant $c_{\delta}$ in (10).

\section{Conclusions}

The experimental results from this study have identified several specific features of the far-field scaling properties of confined, supersonic, planar, turbulent, bluff-body wakes. The classical vortex street-like large-scale structure of incompressible, planar, turbulent wakes is recovered in the far field of the present Mach 2 and Mach 3 wakes at $x$ locations where the local relative Mach number $M_{r}(x)$ has decreased to around 0.4 . Mean velocity profiles in the wake far field are self-similar when scaled by the local flow width and mean centerline velocity deficit, even after the reflected waves from the near-field recompression region interact with the wake. The flow width and mean centerline velocity deficit in the supersonic wakes follow the $\frac{1}{2}$-power law scalings of incompressible planar turbulent wakes in Eqs. (4) and (5), but with changes in $x_{0}, c_{\delta}$, and $c_{u}$. In particular, the virtual origin $\left(x_{0} / \vartheta\right)$ in these scalings shifts downstream in supersonic wakes due to the near-field recompression region. ${ }^{29}$ The changes in $c_{\delta}$ and $c_{u}$ depend on the ability of disturbances produced by the local self-induced forcing of the wake to propagate upstream.

Interactions between the reflected expansion waves originating from the near-field recompression region and the large-scale structures in the wake far field create a local self-induced periodic forcing mechanism that is inherently matched to the local natural frequency of the wake vortices, leading to lock on of the vortical structures to this forcing. The effect is similar to the response of incompressible turbulent mixing layers and wakes to imposed forcing, ${ }^{30-33}$ but in confined supersonic wakes the forcing is self-induced. Because of this self-induced forcing mechanism, the local growth rate and velocity decay rate of the wake increase dramatically immediately downstream of the point where the reflected expansion wave intersects the wake. Sufficiently far downstream of the wave intersection point, the wake returns to its natural growth and velocity decay rates. Upstream of the point where the reflected near-field expansion wave first intersects the wake, the measured far-field scaling constants $c_{\delta}$ and $c_{u}$ of the wake can display either the forced or unforced values from incompressible turbulent wakes, depending on whether the local centerline Mach number $M_{0}(x)$ provides a subsonic path for the wave-induced forcing to propagate upstream. Though the entrainment rate increases dramatically due to this selfinduced forcing, the available evidence (e.g., see Fig. 3) suggests that the mixing rate does not increase. As the wake returns to its natural growth rate, much of the additionally entrained freestream fluid appears to be detrained. This local forcing mechanism and its effects on the wake-scaling properties repeat at each downstream 
location where the reflected expansion waves interesect the wake. The wave interactions with the flow and the resulting self-induced forcing mechanism may represent a stronger effect of compressibility in confined supersonic, planar, turbulent, bluff-body wakes than does any dependence of the scaling constants $c_{\delta}$ and $c_{u}$ on the local relative Mach number $M_{r}(x)$.

\section{Acknowledgments}

This work was supported, in part, by the Air Force Office of Scientific Research (AFOSR) under AFOSR Contracts F49620-951-0115 and F49620-98-1-0003 and by the NASA Constellation University Institutes Project Space Vehicle Technology Institute under Grant NCC3-989, with Claudia Meyer as the Project Manager.

\section{References}

${ }^{1}$ Bogdanoff, D. W., "Compressibility Effects in Turbulent Shear Layers," AIAA Journal, Vol. 21, No. 6, 1982, pp. 926-927.

${ }^{2}$ Chinzei, N., Masuya, G., Komuro, T., Murakami, A., and Kudou, K., "Spreading of Two-Stream Supersonic Turbulent Mixing Layers," Physics of Fluids, Vol. 29, No. 5, 1986, pp. 1345-1347.

${ }^{3}$ Papamoschou, D., and Roshko, A., "The Compressible Turbulent Shear Layer: An Experimental Study," Journal of Fluid Mechanics, Vol. 197, 1988, pp. 453-477.

${ }^{4}$ Samimy, M., and Elliott, G. S., "Effects of Compressibility on the Characteristics of Free Shear Layers," AIAA Journal, Vol. 28, No. 3, 1990, pp. 439-445.

${ }^{5}$ Elliott, G. S., and Samimy, M., "Compressibility Effects in Free Shear Layers," Physics of Fluids A, Vol. 2, No. 7, 1990, pp. 1231-1248.

${ }^{6}$ Dimotakis, P. E., "Turbulent Free Shear Layer Mixing and Combustion," High-Speed Flight Propulsion Systems, edited by S. N. B. Murthy and E. T. Curran, Vol. 137, Progress in Aeronautics and Astronautics, AIAA, Washington, DC, 1991, pp. 265-340.

${ }^{7}$ Goebel, G. S., and Dutton, J. C., "Experimental Study of Compressible Turbulent Mixing Layers," AIAA Journal, Vol. 29, No. 4, 1991, pp. 538-546.

${ }^{8}$ Papamoschou, D., "Structure of the Compressible Turbulent Shear Layer," AIAA Journal, Vol. 29, No. 5, 1991, pp. 680, 681.

${ }^{9}$ Clemens, N. T., "An Experimental Investigation of Scalar Mixing in Supersonic Turbulent Shear Layers," Mechanical Engineering Dept., HTGL Rept. T-274, Stanford Univ., Stanford, CA, 1991

${ }^{10}$ Papamoschou, D., "Effect of Three-Dimensionality on Compressible Mixing," Journal of Propulsion and Power, Vol. 8, No. 1, 1992, pp. 247-249.

${ }^{11}$ Samimy, M., Reeder, M. F., and Elliott, G. S., "Compressibility Effects on Large Structures in Free Shear Flows," Physics of Fluids A, Vol. 4, No. 6, 1992, pp. 1251-1258.

${ }^{12}$ Zeman, O., "Similarity in Supersonic Mixing Layers," AIAA Journal, Vol. 30, No. 5, 1992, pp. 1277-1283.

${ }^{13}$ Clemens, N. T., and Mungal, M. G., "Two- and Three-Dimensional Effects in the Supersonic Mixing Layer," AIAA Journal, Vol. 30, No. 4, 1992, pp. 973-981.

${ }^{14}$ Gruber, M. R., Messersmith, N. L., and Dutton, J. C., "ThreeDimensional Velocity Field in a Compressible Mixing Layer," AIAA Journal, Vol. 31, No. 11, 1993, pp. 2061-2067.

${ }^{15}$ Hall, J. L., Dimotakis, P. E., and Rosemann, H., "Experiments in Nonreacting Compressible Shear Layers," AIAA Journal, Vol. 31, No. 12, 1993, pp. 2247-2254.
${ }^{16}$ Barre, S., Quine, C., and Dussauge, J. P., "Compressibility Effects on the Structure of Supersonic Mixing Layers: Experimental Results," Journal of Fluid Mechanics, Vol. 259, 1994, pp. 47-78.

${ }^{17}$ Elliott, G. S., Samimy, M., and Arnette, S. A., "The Characteristics and Evolution of Large-Scale Structures in Compressible Mixing Layers," Physics of Fluids A, Vol. 7, No. 4, 1995, pp. 864-876.

${ }^{18}$ Clemens, N. T., and Mungal, M. G., "Large-Scale Structure and Entrainment in the Supersonic Mixing Layer," Journal of Fluid Mechanics, Vol. 284, 1995, pp. 171-216.

${ }^{19}$ Freund, J. B., Lele, S. K., and Moin, P., "Compressibility Effects in a Turbulent Annular Mixing Layer 1. Turbulence and Growth Rate," Journal of Fluid Mechanics, Vol. 421, 2000, pp. 229-267.

${ }^{20}$ Freund, J. B., Lele, S. K., and Moin, P., "Compressibility Effects in a Turbulent Annular Mixing Layer. Part 2. Mixing of a Passive Scalar," Journal of Fluid Mechanics, Vol. 421, 2000, pp. 269-292.

${ }^{21}$ Smith, K. M., and Dutton, J. C., "Investigation of Large-Scale Structures in Supersonic Planar Base Flows," AIAA Journal, Vol. 34, No. 6, 1996, pp. $1146-1152$.

${ }^{22}$ Mathur, T., and Dutton, J. C., "Velocity and Turbulence Measurements in a Supersonic Base Flow with Mass Bleed," AIAA Journal, Vol. 34, No. 6, 1996, pp. 1153-1159.

${ }^{23}$ Boswell, B. A., and Dutton, J. C., "Velocity Measurements in a ThreeDimensional Compressible Base Flow," AIAA Journal, Vol. 41, No. 5, 2003, pp. 785-794.

${ }^{24}$ Kastengren, A. L., and Dutton, J. C., "Large-Structure Topology in a Three-Dimensional Supersonic Base Flow," AIAA Journal, Vol. 43, No. 5, 2005, pp. 1053-1063.

${ }^{25}$ Kuehner, J. P., and Dutton, J. C., "Planar Fluorescence Imaging of a Supersonic Axisymmetric Base Flow with Mass Bleed," AIAA Journal, Vol. 43, No. 8, 2005, pp. 1767-1775.

${ }^{26}$ Amatucci, V. A., Dutton, J. C., Kuntz, D. W., and Addy, A. L., "TwoStream, Supersonic, Wake Flow Field Behind a Thick Base. I. General Features," AIAA Journal, Vol. 30, No. 8, 1992, pp. 2039-2046.

${ }^{27}$ Clemens, N. T., and Mungal, M. G., "A Planar Mie Scattering Technique for Visualizing Supersonic Mixing Flows," Experiments in Fluids, Vol. 11, 1991, pp. 175-185.

${ }^{28}$ Wygnanski, I., Champagne, F., and Marasli, B., "On the Large-Scale Structures in Two-Dimensional, Small-Deficit, Turbulent Wakes," Journal of Fluid Mechanics, Vol. 168, 1986, pp. 31-71.

${ }^{29}$ Nakagawa, M., and Dahm, W. J. A., "The Virtual Origin of Subsonic and Supersonic Planar Turbulent Bluff-Body Wakes," AIAA Journal, Vol. 43, No. 3, 2005, pp. 697-700.

${ }^{30}$ Marasli, B., Champagne, F. H., and Wygnanski, I., "Effect of Travelling Waves on the Growth of a Plane Turbulent Wake," Journal of Fluid Mechanics, Vol. 235, 1992, pp. 511-528.

${ }^{31}$ Oster, D., and Wygnanski, I., "The Forced Mixing Layer Between Parallel Streams," Journal of Fluid Mechanics, Vol. 123, 1982, pp. $91-130$

${ }^{32}$ Roberts, F. A., "Effects of a Periodic Disturbance on Structure and Mixing in Turbulent Shear Layers and Wakes," Ph.D. Dissertation, Aeronautics Dept., California Inst. of Technology, Pasadena, CA, 1985.

${ }^{33}$ Roberts, F. A., and Roshko, A., "Effects of Periodic Forcing on Mixing in Turbulent Shear Layers and Wakes," AIAA Paper 85-0570, 1985.

W. Ng 